\title{
Gonorrhea treatment guidelines in Canada: 2004 update
}

$\mathrm{R}_{\mathrm{a}}$ eaders of $C M A 7$ were first alerted to the rising incidence of ciprofloxacin-resistant Neisseria gonorrboeae infection in Canada last year. ${ }^{1}$ On the basis of this trend, the recommended treatment for gonorrhea in some areas of Canada and for some infected patients has changed.

The reported rate of gonorrhea in Canada reached its lowest point in 1997 , but since that year, following more than 2 decades of decline, the rate has increased by over $60 \%$ to 24.3 per 100000 (7363 cases) in 2003 (preliminary unpublished data from the Sexual Health and STI Section, Public Health Agency of Canada).

Gonorrhea is caused by $N$. gonorrboeae infection, and common clinical manifestations include urethritis and cervicitis. Uncomplicated cases of gonorrhea can be treated with single-dose antimicrobial therapy, which helps to ensure compliance. However, emerging and prevalent antibiotic-resistant strains of $N$. gonorrboeae limit therapeutic choices. Penicillin- and tetracycline-resistant strains of $N$. gonorrboeae are well documented around the world, and therefore penicillin and tetracycline are not recommended for the treatment of gonorrhea. Fluoroquinolone (FQ) resistance was first identified in 1992. FQ resistance is currently most prevalent in the Far East, but it has been documented in many parts of the world, including the UK, the United States and Canada. ${ }^{2}$

Once resistance rates to an antibiotic reach 3\%-5\%, the antibiotic can no longer be recommended. Box 1 lists the areas and populations for which FQs are not recommended for treatment of gonorrhea.

Preliminary national FQ resistance rates in Canada have been reported to be $2.1 \%$ in 2002 and $2.0 \%$ in 2003 (unpublished data, National Microbiology Laboratory). These rates are calculated using the number of $N$. gonorrboeae isolates that test resistant to ciprofloxacin (minimum inhibitory concentration $\geq 1.0 \mathrm{mg} / \mathrm{L}$ ) as the numerator with the total number of $N$. gonorrboeae isolates tested each year as the denominator. Before 1997 the number of isolates tested by the NML was close to the total number of national gonorrhea cases reported; however, this is no longer the case. In 2003, the number of isolates submitted for antibiotic resistance testing nationally was 4726 , and the preliminary number of gonorrhea cases reported nationally was 7363. The drop in national resistance testing can be explained at least in part by the growing use of diagnostic methods other than culture, such as nucleic acid amplification testing (e.g., polymerase chain reaction and ligase chain reaction), which preclude antimicrobial resistance testing.

Although the national rate remained under $3 \%$ in 2003 , data on FQ resistance from individual provinces and territories indicate that the rate of resistance is actually much higher in some jurisdictions. Some provincial laboratories are reporting FQ resistance rates of at least $10 \%$ for those isolates submitted for testing.

There are 4 recommended treatments in Canada for uncomplicated urethral, endocervical, rectal and pharyngeal infections (Box 2). Ciprofloxacin and ofloxacin should not be used in cases where the risk of FQ resistance is significant (Box 1). It is important to note that regions within Canada and the US are included in this list. Physicians in Canada who are treating gonorrhea are advised to seek information, if available, from local public health authorities about the level of FQ resistance in their area. If the risk of FQ resistance is significant, the recommended treatments are limited to cefixime or ceftriaxone.

If allergy precludes the use of cephalosporins, treatment recommendations include azithromycin or spectinomycin. A $2-\mathrm{g}$ dose of azithromycin is associated with a significant incidence of gastrointestinal adverse effects. Taking the tablet with food may minimize such adverse effects. Antiemetics may be needed. In Canada, spectinomycin is available only through the Special Access Programme, Health Canada.

If because of tolerance or access issues azithromycin and spectinomycin cannot be used, and the case occurs in an area of significant FQ resistance, ciprofloxacin or ofloxacin may be considered for patients with allergies to cephalosporins. However, FQs may be used in this situation only if treatment is followed by a test of cure, and they are acceptable only for patients who are likely to return for follow-up testing.

The recommended treat-

Box 1: Areas and populations for which fluoroquinolones (FQs) are not recommended for the treatment of gonorrhea
Areas
- Asia
- Pacific Islands (including Hawaii)
- India
- Israel
- Australia
- United Kingdom
- United States: California, Washington State, Arizona (Maricopa County), Michigan (Ingham, Clinton, Eaton, Jackson, Livingston and Shiawassee counties)
- Areas in Canada experiencing rates of FQ resistance greater than 3\%-5\%: check with local public health officials to learn about FQ resistance in your area
- Any area with rates of FQ-resistant N. gonorrhoeae greater than $3 \%-5 \%$

\section{Populations}
- Men who have sex with men who are epidemiologically linked to the US
- People with sexual contacts from the areas listed above 
ments for pregnant or nursing women and for children under the age of 9 remain unchanged. Physicians are referred to the 1998 Canadian STD Guidelines, available online at www. hc-sc.gc.ca/pphb-dgspsp/publicat /std-mts98/index.html.

Antibiotic-resistant strains of $N$ gonorrboeae continue to challenge the treatment, prevention and control of this infection. Because of the availability and use of alternative testing methods that do not allow resistance testing, ongoing monitoring and reporting of antimicrobial resistance remains vital. As well, enhanced patient information beyond age and sex (e.g., whether the infection was derived from homosexual or heterosexual contact) would enable better targeting of health promotion strategies and public education campaigns. As such, a national sentinel surveillance system for the ongoing monitoring of resistance of $N$. gonorrhoeae to various antimicrobials, such as those in place in the UK and US, may need to be introduced in Canada.

For further updates on FQresistant $N$. gonorrboeae in Canada, you may access the Sexual Health and STI Section Web site on the Public Health Agency

Box 2: Recommended treatment of urethral, endocervical, rectal or pharyngeal gonorrhea in patients 9 years of age and older (except pregnant or nursing mothers)

If there is no suspected resistance to FQs and patient has no cephalosporin allergy or history of immediate or anaphylactic reactions to penicillin
Cefixime
$400 \mathrm{mg}$ orally in single dose
Ceftriaxone*
$125 \mathrm{mg}$ intramuscularly in
single dose
Ciprofloxacin $†$
$500 \mathrm{mg}$ orally in single dose
Ofloxacint
$400 \mathrm{mg}$ orally in single dose

If use of FQs is not recommended

Cefixime $\quad 400 \mathrm{mg}$ orally in single dose

Ceftriaxone* $125 \mathrm{mg}$ intramuscularly in

single dose

If use of FQs is not recommended and patient has cephalosporin allergy or history of immediate or anaphylactic reactions to penicillin
Azithromycin $\ddagger$
$2 \mathrm{~g}$ orally in single dose
Spectinomycin§
$2 \mathrm{~g}$ intramuscularly in single dose (available only
through Special Access Programme)

If use of FQs is not recommended but all other treatments are not tolerated or available, FQs may be used. Treatment with FQs must be followed by a test of cure and is acceptable only for patients who are likely to present for follow-up testing

\section{Ciprofloxacint \\ $500 \mathrm{mg}$ orally in single dose \\ Ofloxacin† \\ $400 \mathrm{mg}$ orally in single dose}

*The preferred diluent for ceftriaxone is $1 \%$ lidocaine without epinephrine $(0.9 \mathrm{~mL} / 250 \mathrm{mg}, 0.45$ $\mathrm{mL} / 125 \mathrm{mg}$ ) to reduce discomfort.

†Ciprofloxacin and ofloxacin are contraindicated in pregnant and nursing women. The safety of FQs in children has not been established. Articular damage has been observed in studies of young animals exposed to FQs, although this has not been shown to date in humans. FQs should not be used in prepubertal children. Clinical judgement should be exercised when considering FQ use in postpubertal adolescents under the age of 18 .

¥A 2-g dose of azithromycin is associated with a significant incidence of gastrointestinal adverse effects. Taking the tablet with food may minimize such adverse effects. Antiemetics may be needed.

Slf spectinomycin is used, a test of cure is recommended. Spectinomycin should not be used to treat pharyngeal infections.

of Canada Web site at: www.hcsc.gc.ca/pphb-dgspsp/std-mts/.

To ensure the effective and appropriate treatment of gonorrhea in Canada, an Interim Statement on the Treatment of Gonorrhea in Canada has been produced and will be available on the Public Health Agency of Canada Web site at www.phac-aspc .gc.ca. The revised version of the Canadian STD Guidelines is targeted for release in autumn 2005 and will reflect the changes that have occurred in FQ resistance of $N$. gonorrhoeae since the 1998 edition.

\section{Janice Mann}

Rhonda Kropp

Tom Wong

Sexual Health and STI Section

Community Acquired Infections Division

Centre for Infectious Disease

Prevention and Control

Public Health Agency of Canada

Ottawa, Ont.

Sylvie Venne

Services des maladies infectieuses

Direction générale de la santé

publique

Ministère de la Santé et des Services sociaux, Québec

Montréal, Qué.

Barbara Romanowski

Division of Infectious Diseases

Faculty of Medicine and Dentistry

University of Alberta

Edmonton, Alta.

Acknowledgements: The treatment recommendations in this article were made in conjunction with the Expert Working Group for the Canadian STI Guidelines and the provincial and territorial STI Directors. We thank Lai King $\mathrm{Ng}$ and Mei Ling Lam from the National Microbiology Laboratory.

\section{References}

1. Sarwal S, Wong T, Sevigny C, Ng LK Increasing incidence of ciprofloxacinresistant Neisseria gonorrboeae infection in Canada. CMAJ 2003;168(7): 872-3.

2. Ng LK, Sawatzky P, Martin IE, Booth S. Characterization of ciprofloxacin resistance in Neisseria gonorrhoeae isolates in Canada. Sex Trans Dis 2002;29(12):780-8.

3. Increases in fluoroquinolone-resistant Neisseria gonorrboeae among men who have sex with men - United States, 2003 , and revised recommendations for gonorrhea treatment, 2004. MMWR Morb Mortal Wkly Rep 2004; 53(16):335-8. 